\title{
Treating restenosis after drug-eluting stent implantation
}

Drug-eluting stents (DES), including stents eluting sirolimus or one of its analogues (limus-eluting stents), are a common choice of treatment for patients with coronary artery disease. Although these stents are superior to bare-metal stents, some patients with DES will eventually have restenosis. Repeat stenting is an option for these patients, but some clinicians are concerned about the potential effects of several stent layers in the vessel wall. The ISAR-DESIRE 3 study investigators have now shown that paclitaxel-eluting balloons (PEB) are not inferior to paclitaxel-eluting stents (PES) for the treatment of patients with restenosis. "The prospect of achieving high antirestenotic efficacy without requirement for additional stent implantation

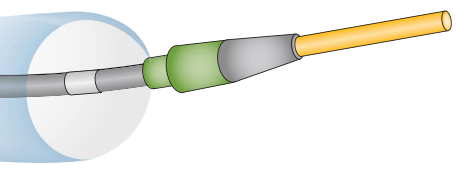

is intuitively attractive," says Robert Byrne, lead author on the study report.

The investigators enrolled 402 patients with restenosis after implantation of a limus-eluting stent and randomly assigned them to receive a PES $(n=131), \mathrm{PEB}$ $(n=137)$, or balloon angioplasty with no drug elution $(n=134)$. Paclitaxel was used in both drug-eluting devices to restrict differences to the device itself. PES and PEB were superior to angioplasty with a noneluting balloon, as measured by the diameter of stenosis 6-8 months after treatment $(37.4 \%, 38.0 \%$, and $54.1 \%$ respectively). PEB was noninferior to PES.

Target lesion revascularization 1 year after the procedure occurred less frequently in patients treated with PES or PEB than in patients who had angioplasty with a noneluting balloon $(13.5 \%, 22.1 \%$, and $43.5 \%$ respectively). The differences between PES and PEB were not statistically significant. All groups had comparable rates of death, myocardial infarction and target lesion thrombosis.
In their study report, the researchers highlight that trials involving longer follow-up in larger groups of patients are needed to confirm the safety of using PEB in this setting and to determine whether any differences in the effects of PEB and PES are clinically meaningful. They also point out that their findings might not be applicable to other drug-eluting balloon catheters.

The researchers have already begun the ISAR-DESIRE 4 study, which will examine whether the efficacy of PEB treatment could be improved by first preparing the lesion with cutting or scoring balloon dilation. "Among other benefits, such an approach might improve tissue drug distribution," says Byrne.

Megan Cully

Original article Byrne, R. A. et al. Paclitaxel-eluting balloons, paclitaxel-eluting stents, and balloon angioplasty in patients with restenosis after implantation of a drugeluting stent (ISAR-DESIRE 3): a randomised, open-label trial. Lancet doi:10.1016/S0140-6736(12)61964-3 